\title{
A new lupane-type triterpenoid fatty acid ester and other isolates from Ophiorrhiza shendurunii
}

Renjith Rajan ${ }^{\mathrm{a}}$, Ramaswamy Venkataraman ${ }^{\mathrm{b}}$ and Sabulal Baby,

${ }^{a}$ Phytochemistry and Phytopharmacology Division, Jawaharlal Nehru Tropical Botanic Garden and Research Institute, Pacha-Palode, Thiruvananthapuram 695 562, Kerala, India

${ }^{b}$ Department of Chemistry, Sri Paramakalyani College, Manonmaniom Sundaranar University, Tirunelveli, Alwarkurichi 627 412, Tamil Nadu, India

Dr. Sabulal Baby (E-mail: sabulal@ gmail.com; Tel: +91-472-2869226 ext. 214; Fax: +91-4722869646)

\begin{abstract}
A new pentacyclic triterpenoid fatty acid ester, lupan-20-ol-3( $\beta$ )-yl hexadecanoate (1), together with lupan-20-ol-3( $\beta$ )-yl acetate (2), olean-18-en-3( $\beta$ )-yl hexadecanoate $(3)$, dotriacontanoic acid (4), stigmasterol (5), rubiadin (6), nonadecanoic acid (7), palmitic acid (8) and camptothecin (9) were isolated from the hexane and chloroform extracts of Ophiorrhiza shendurunii from south India. Structures of the isolates were determined by ${ }^{1} \mathrm{H}-,{ }^{13} \mathrm{C}-,{ }^{13} \mathrm{C}-\mathrm{DEPT},{ }^{1} \mathrm{H}-{ }^{1} \mathrm{H} \mathrm{COSY}, \mathrm{HMBC}$, HSQC, NOESY NMR, FT-IR, DART-MS, ESI-MS, alkaline hydrolysis, derivatization, GC-MS and HPTLC analyses. O. shendurunii hexane and chloroform extracts showed significant activities against Candida albicans and Fusarium oxysporum. Compounds $\mathbf{1}$ to $\mathbf{3}$ showed only moderate antiyeast/antifungal activities.
\end{abstract}

Keywords: Ophiorrhiza shendurunii, pentacyclic triterpenoid fatty acid ester, lupan-20-ol-3( $\beta$ )yl hexadecanoate, antifungal activity. 
Fig. S1 Spectral data of Lupan-20-ol-3( $\beta$ )-yl hexadecanoate (1)

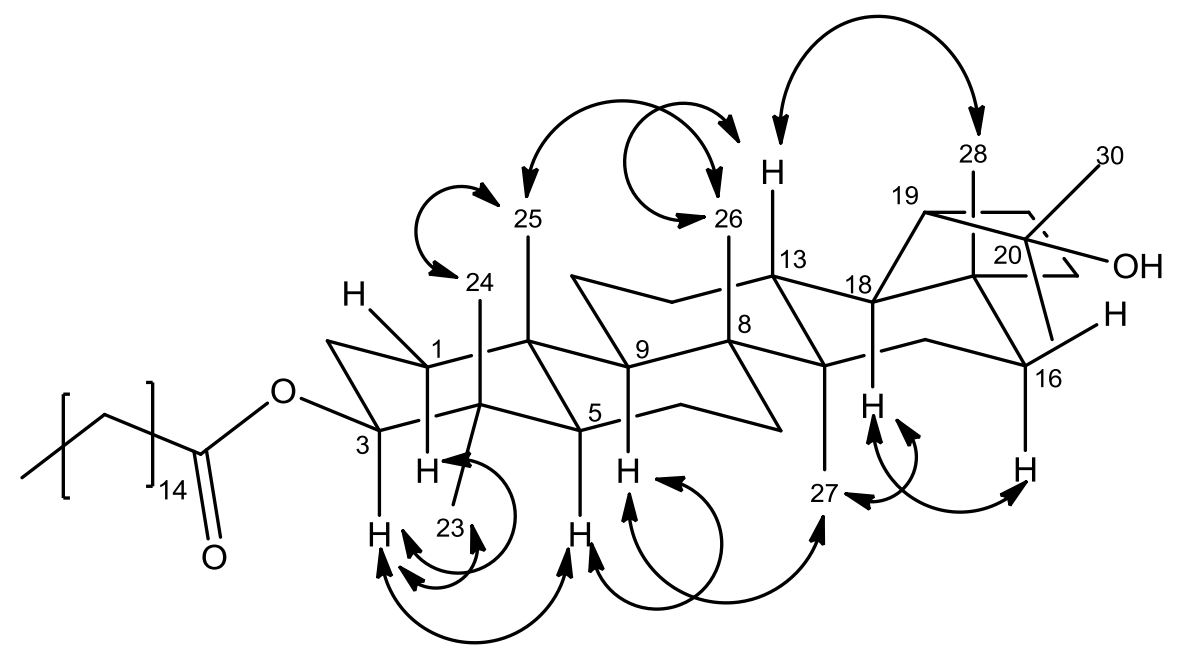

Key NOESY correlations of lupan-20-ol-3( $\beta$ )-yl hexadecanoate (1). 


\section{${ }^{1} \mathrm{H}$ NMR}

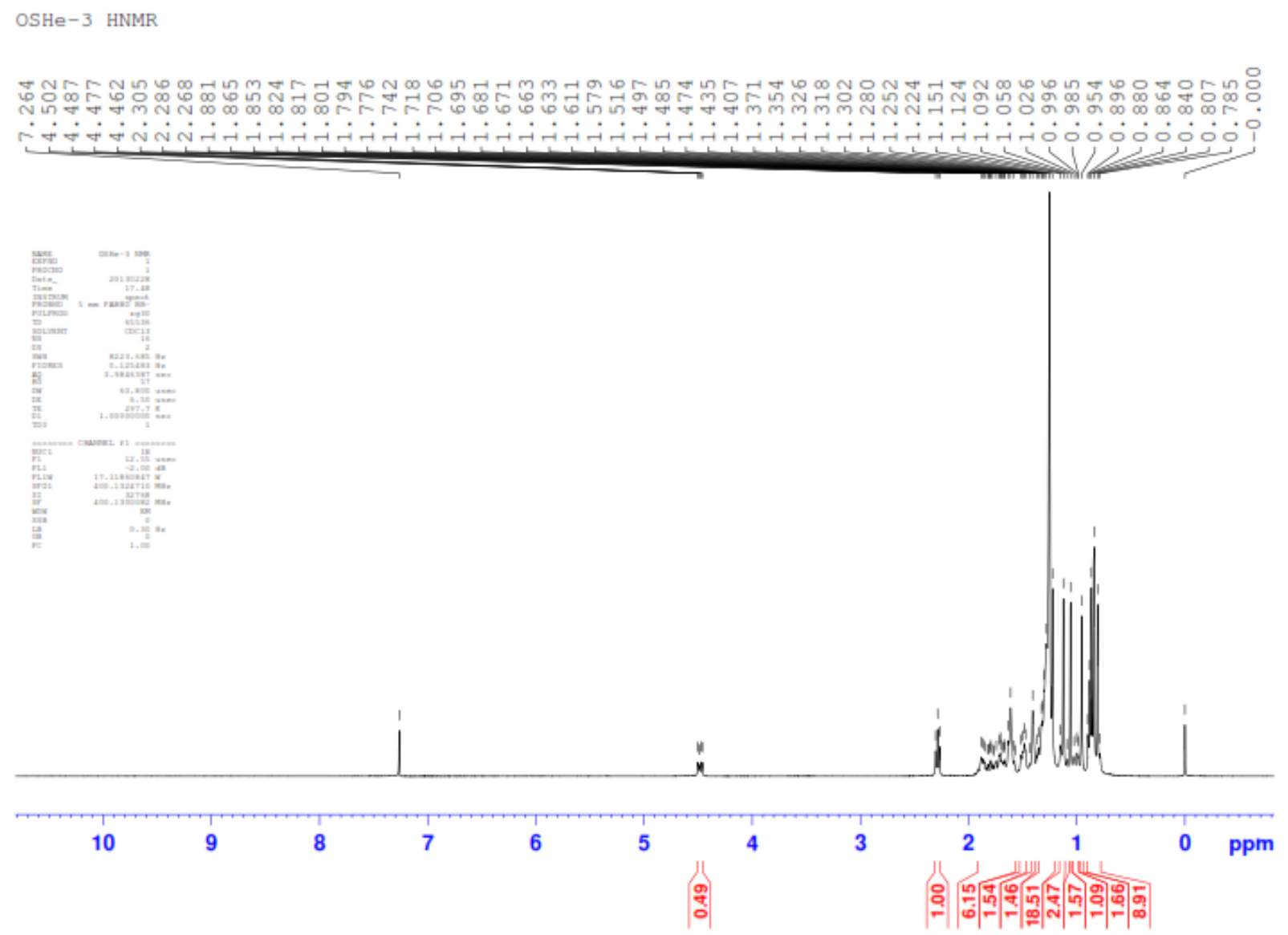




\section{${ }^{1}$ H NMR EXPANSION 01}

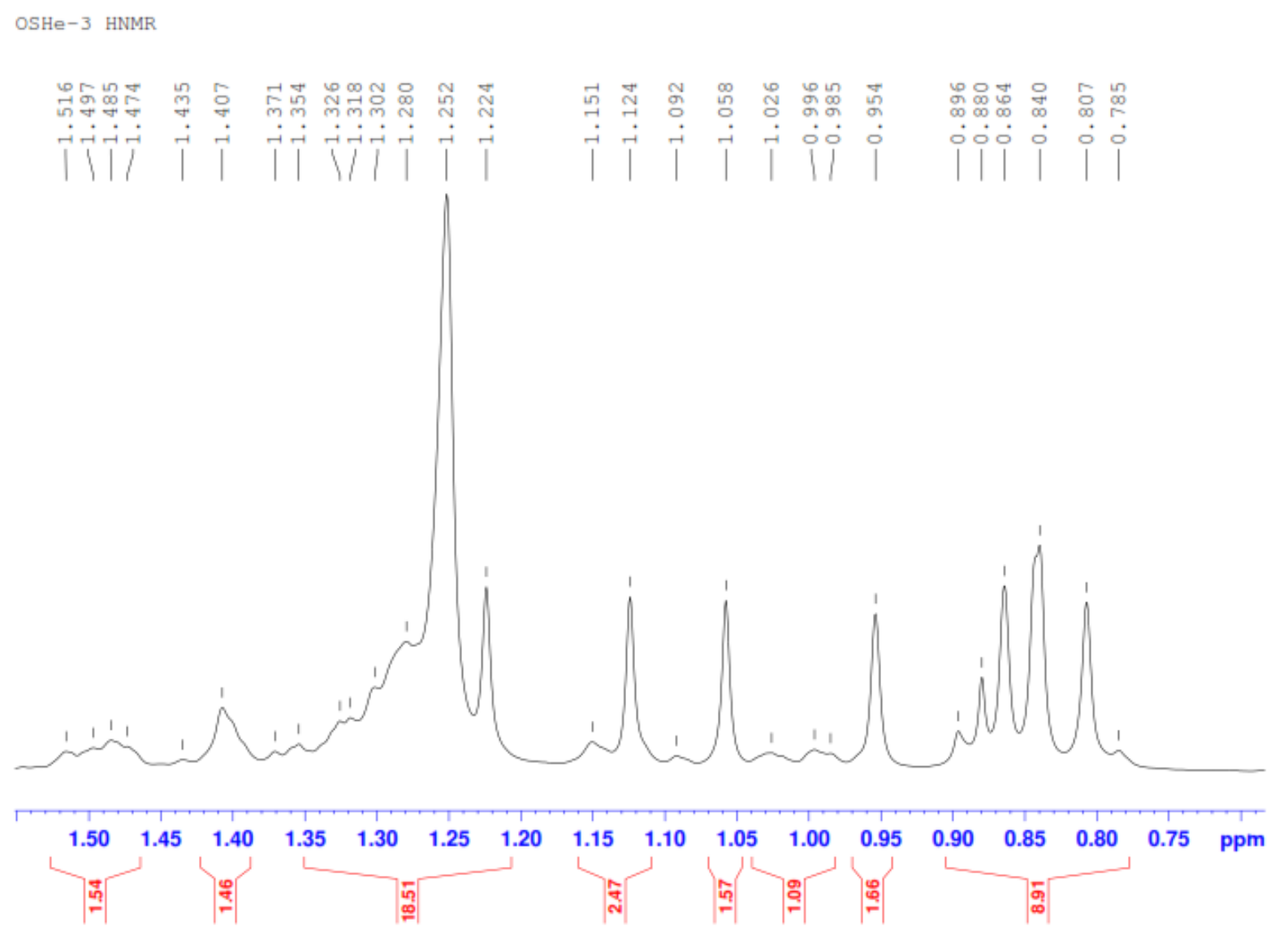




\section{${ }^{13} \mathrm{C}$ NMR}

$$
\text { OSHe-3 CNMR }
$$
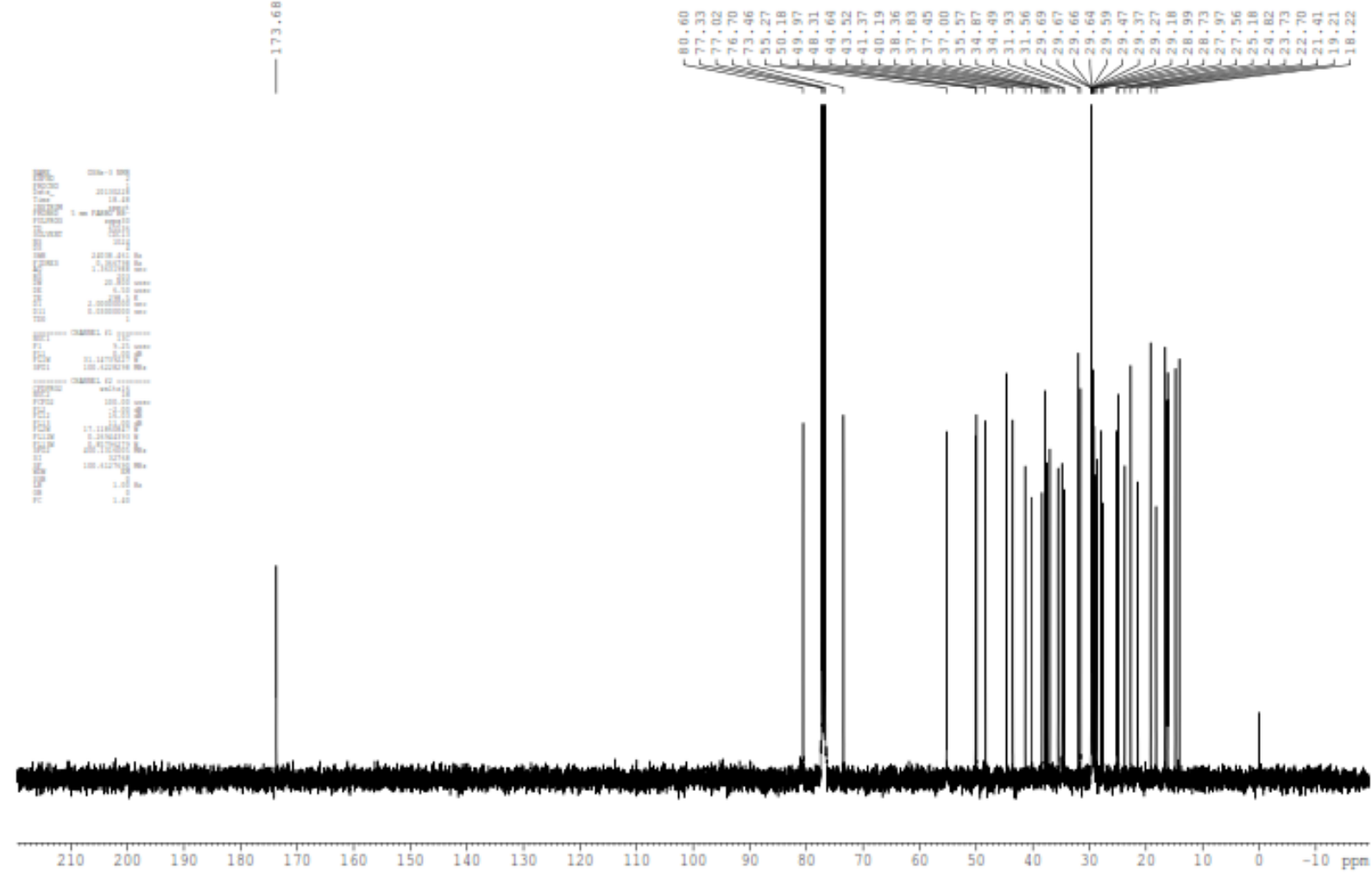


\section{${ }^{13}$ C NMR EXPANSION 01}

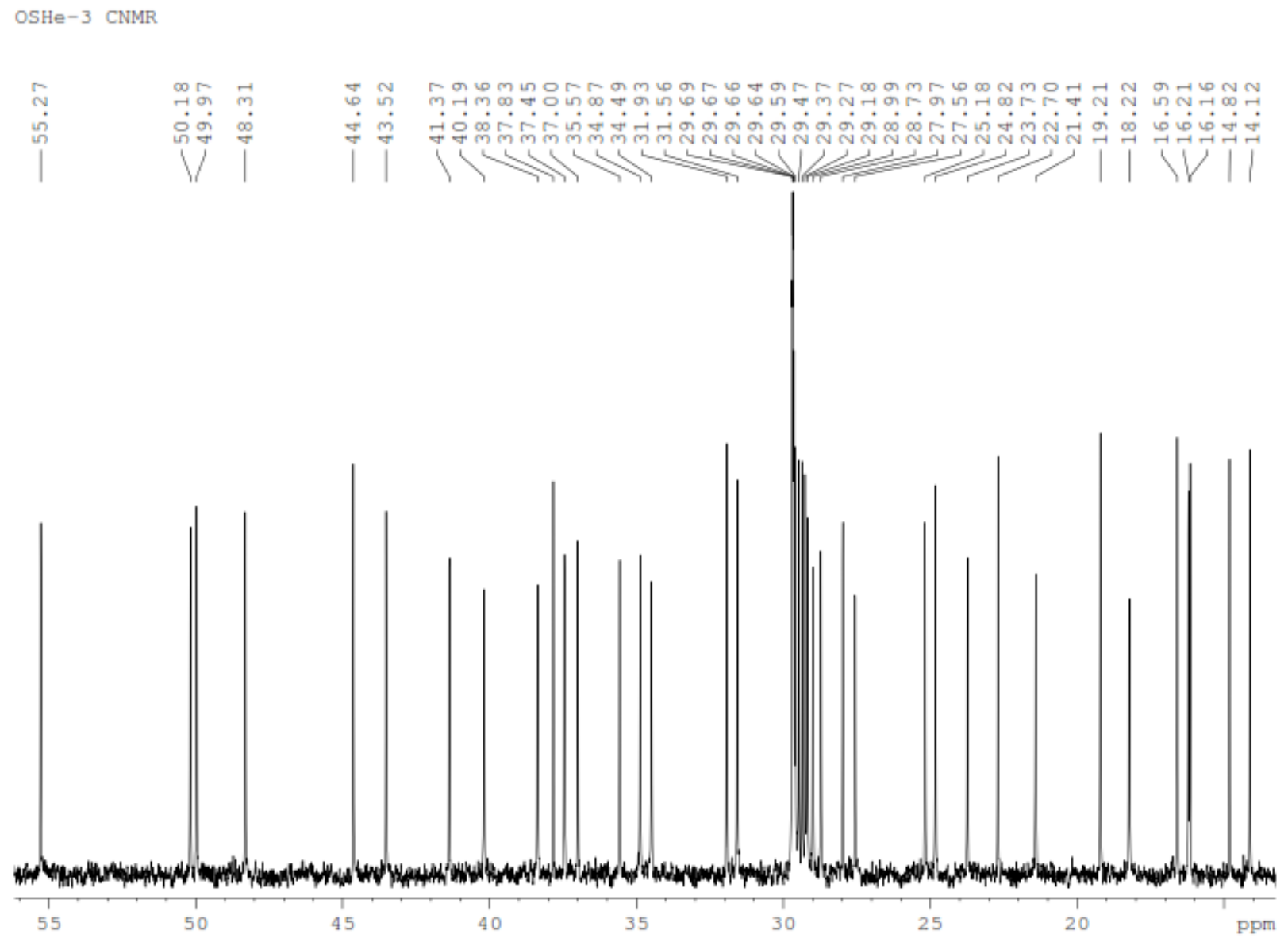




\section{${ }^{13}$ C NMR EXPANSION 02}

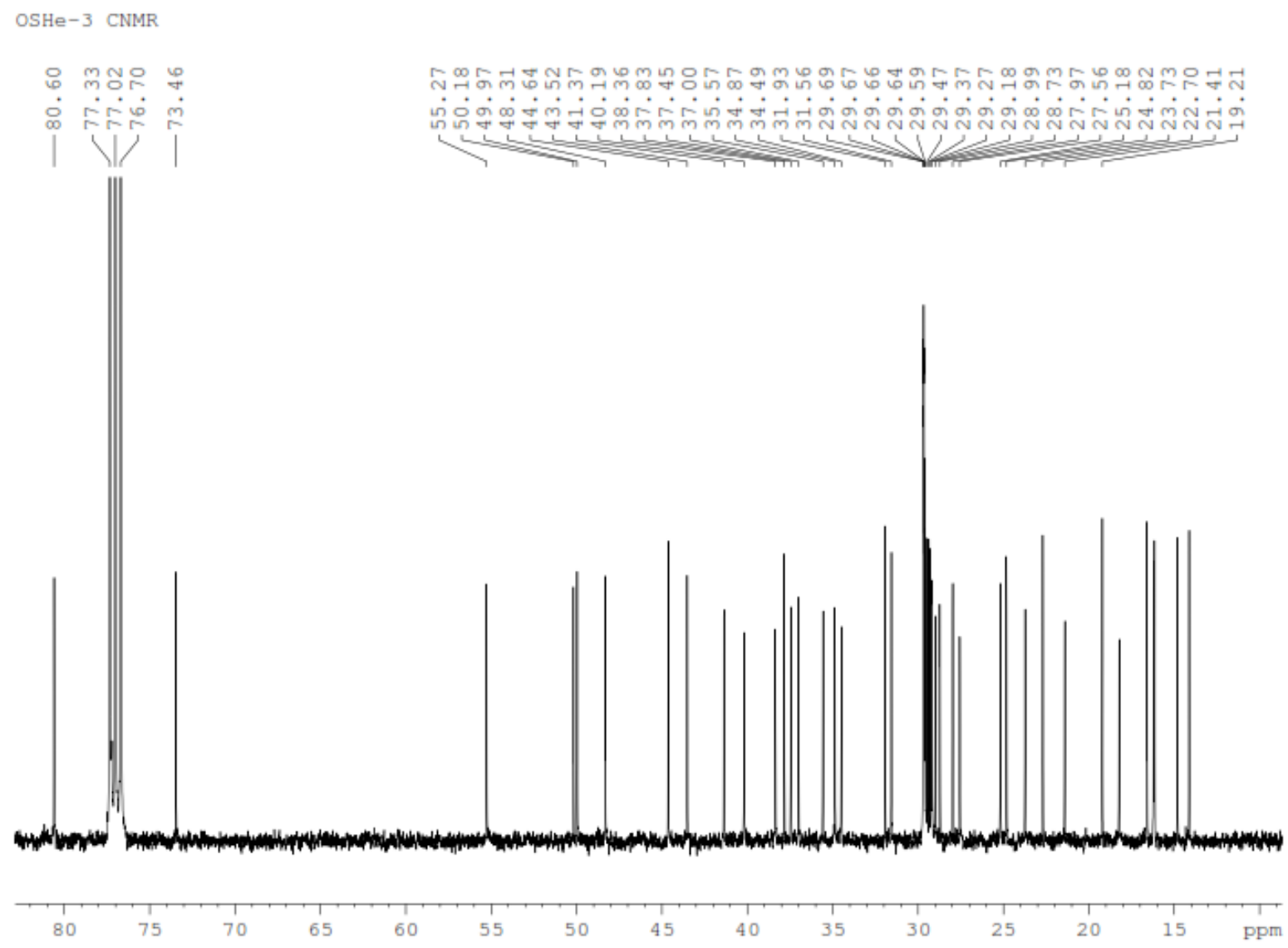




\section{${ }^{13}$ C NMR EXPANSION 03}

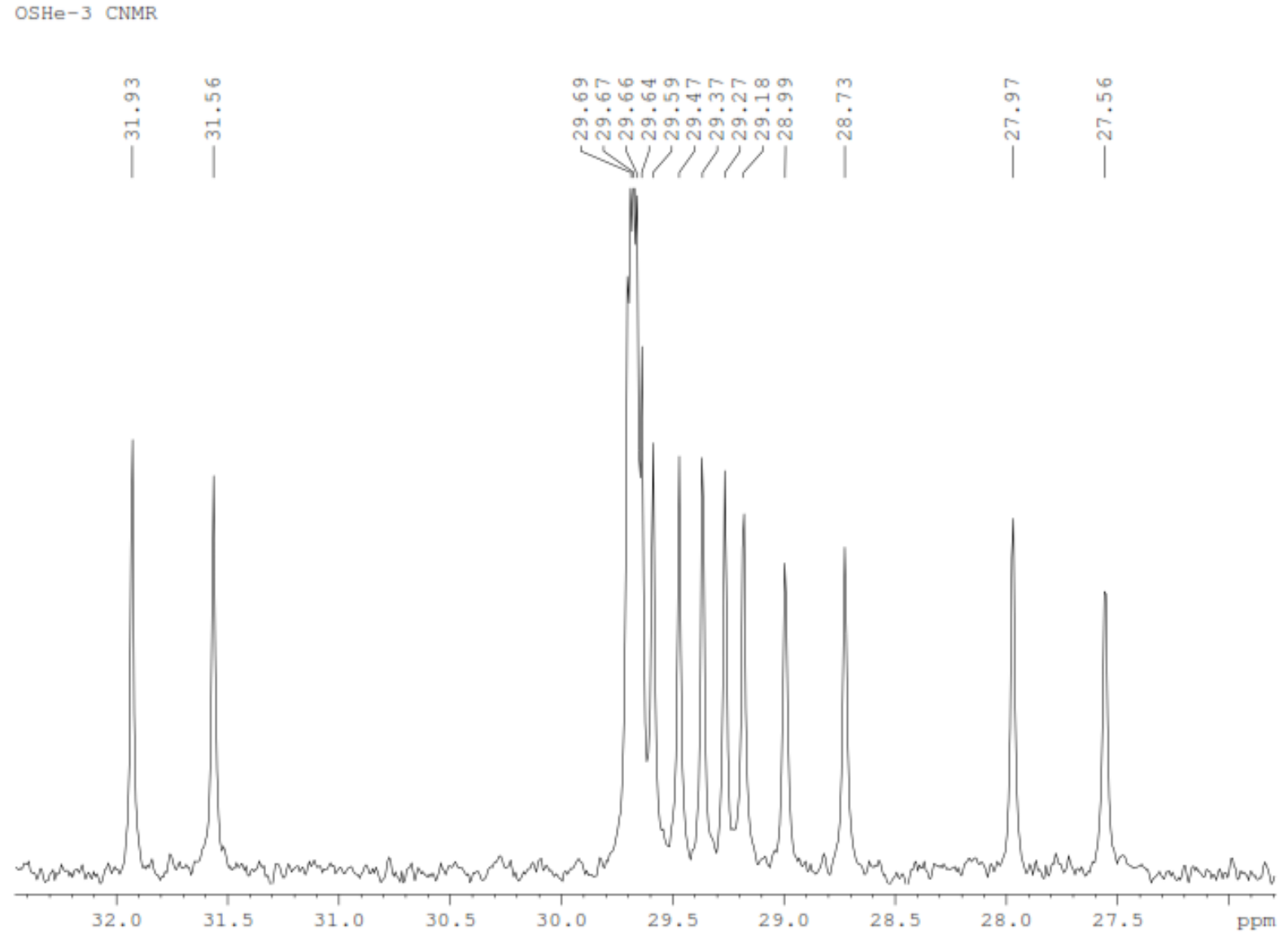




\section{DEPT 90}

OSHe-3 DEPT9O

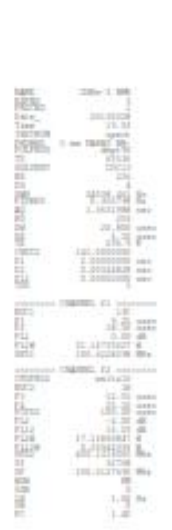

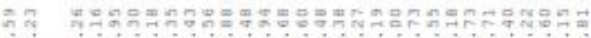

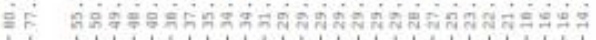

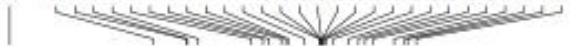

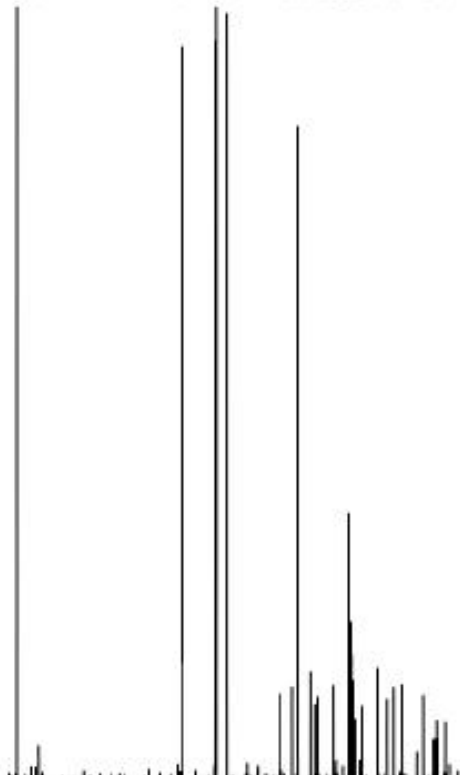

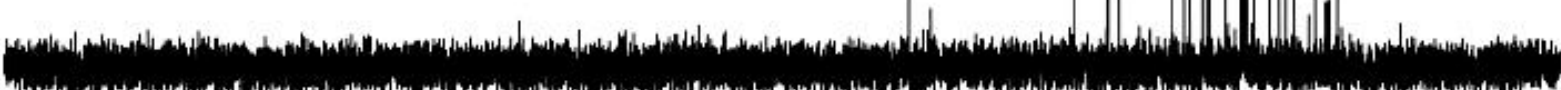

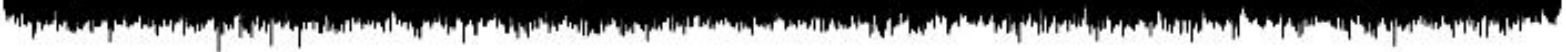

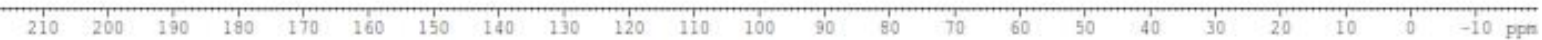




\section{DEPT 135}

osHe-3 DEPT135

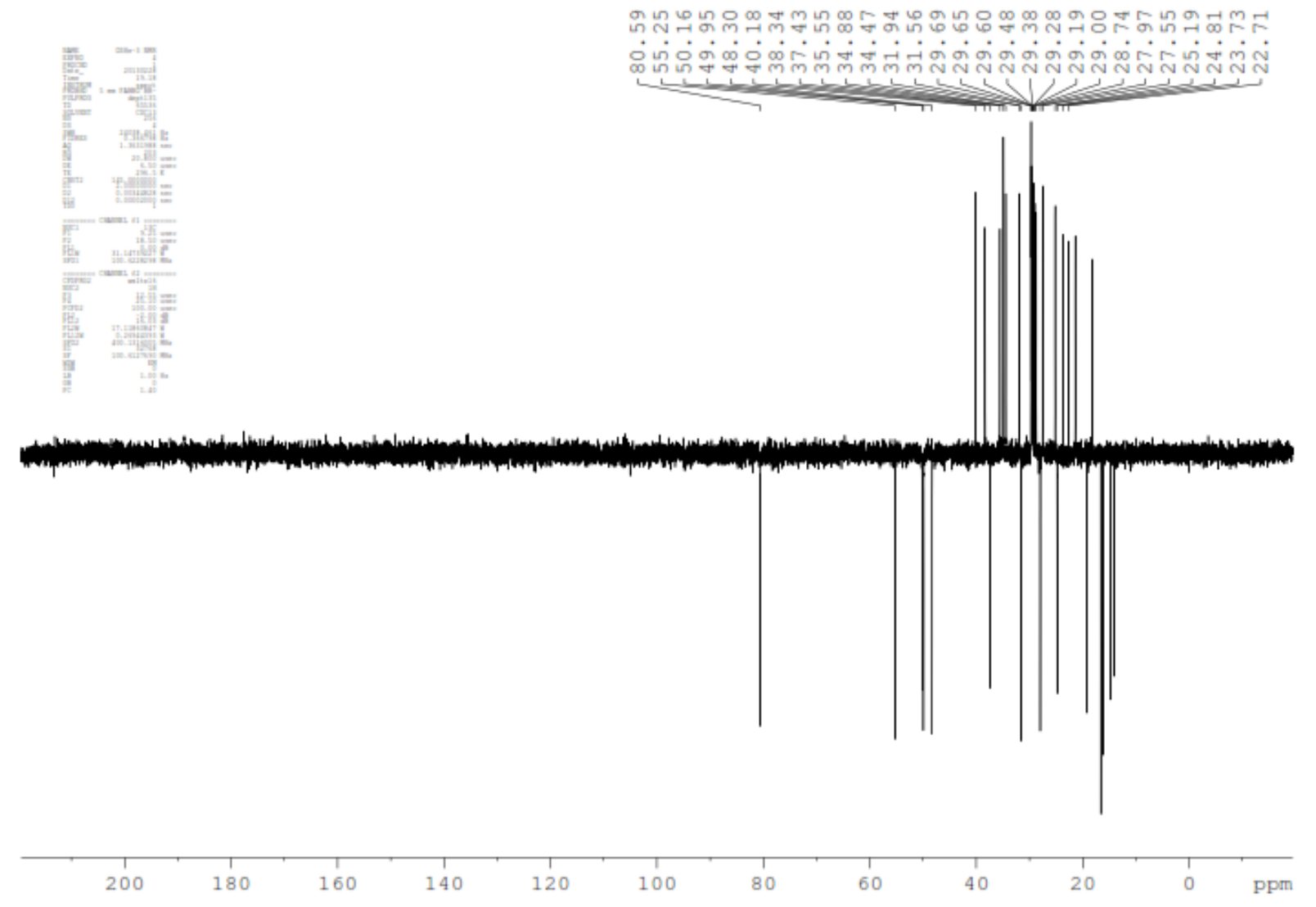




\section{DEPT 135 EXPANSION 01}

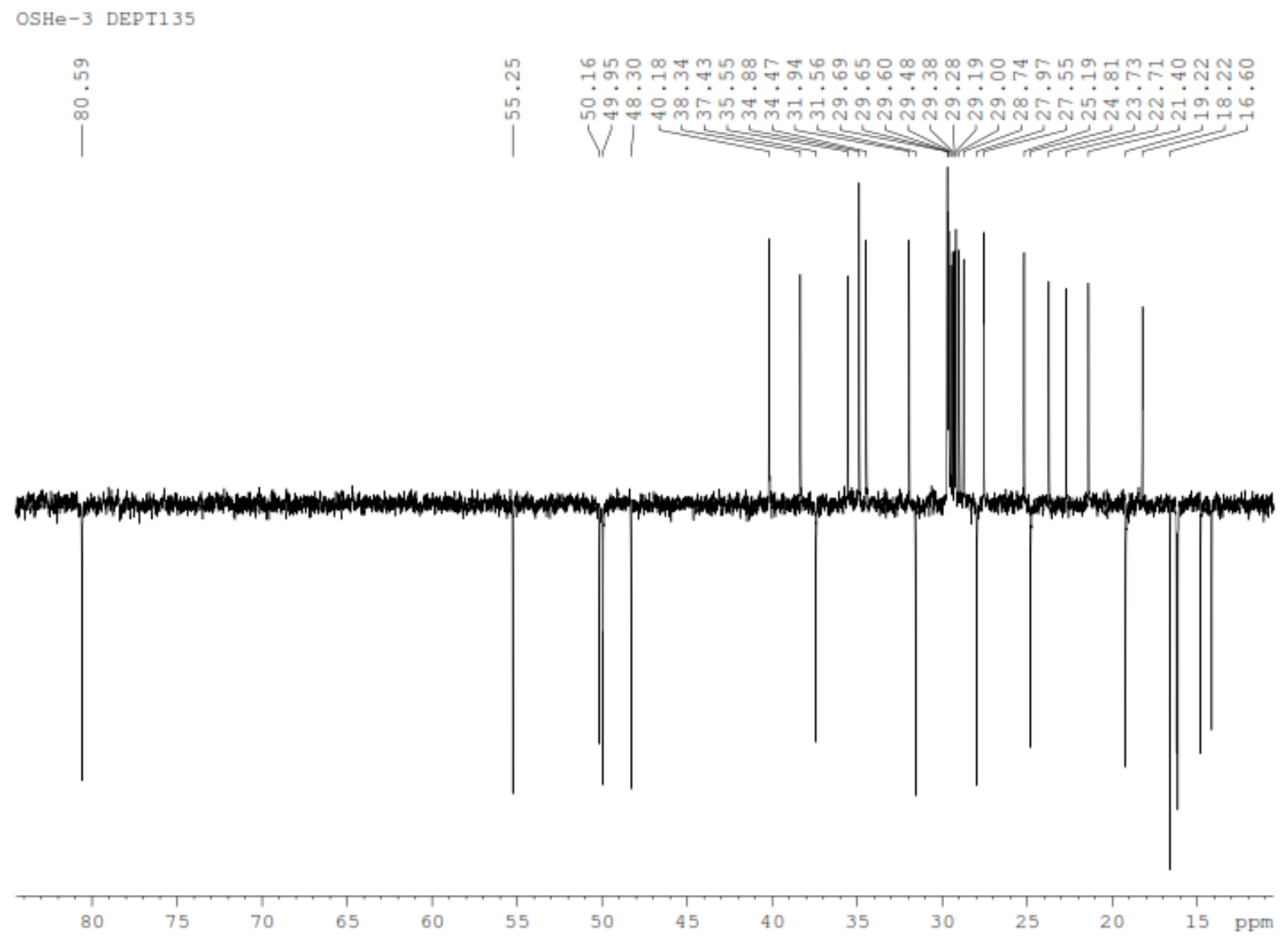


COSY

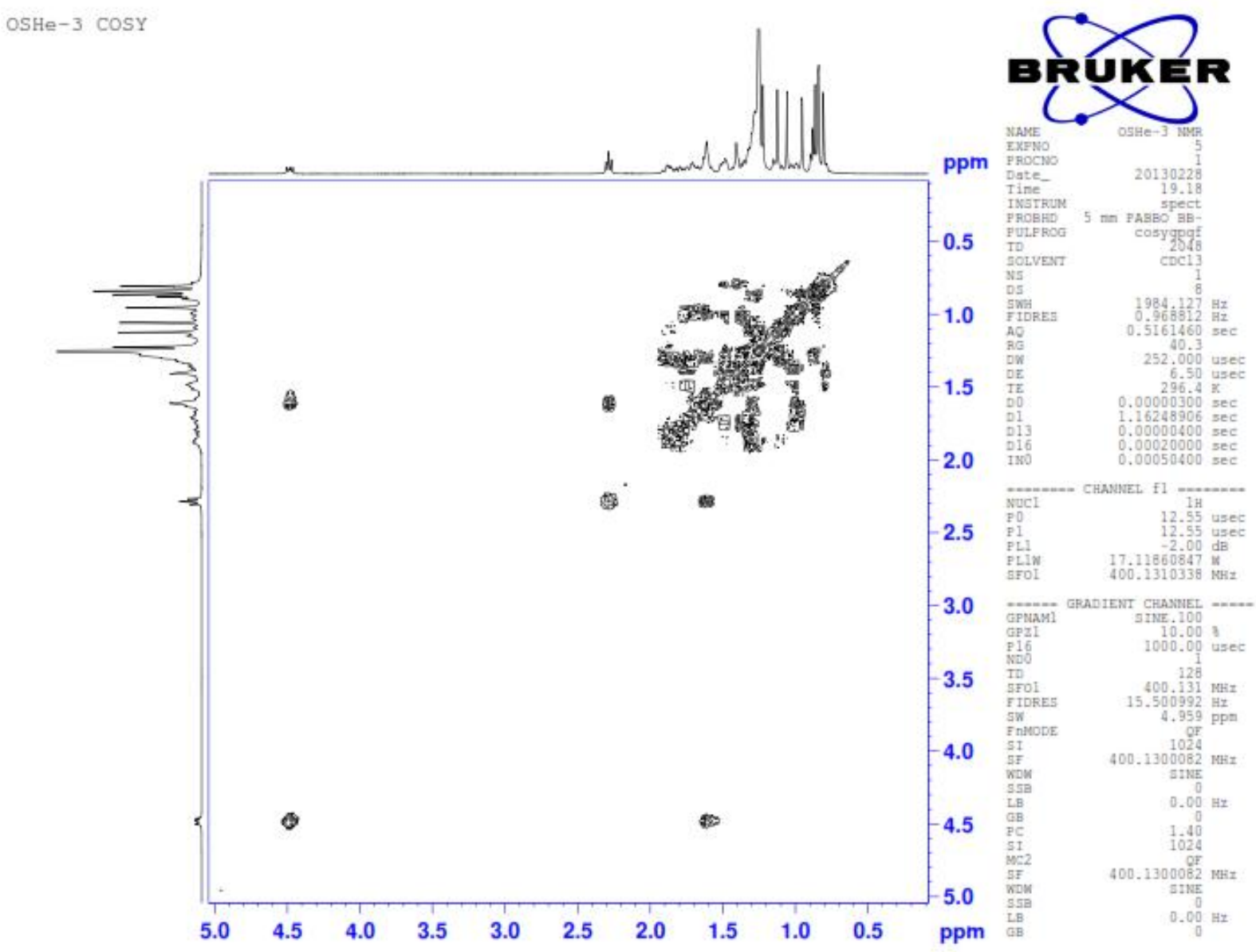


HMBC

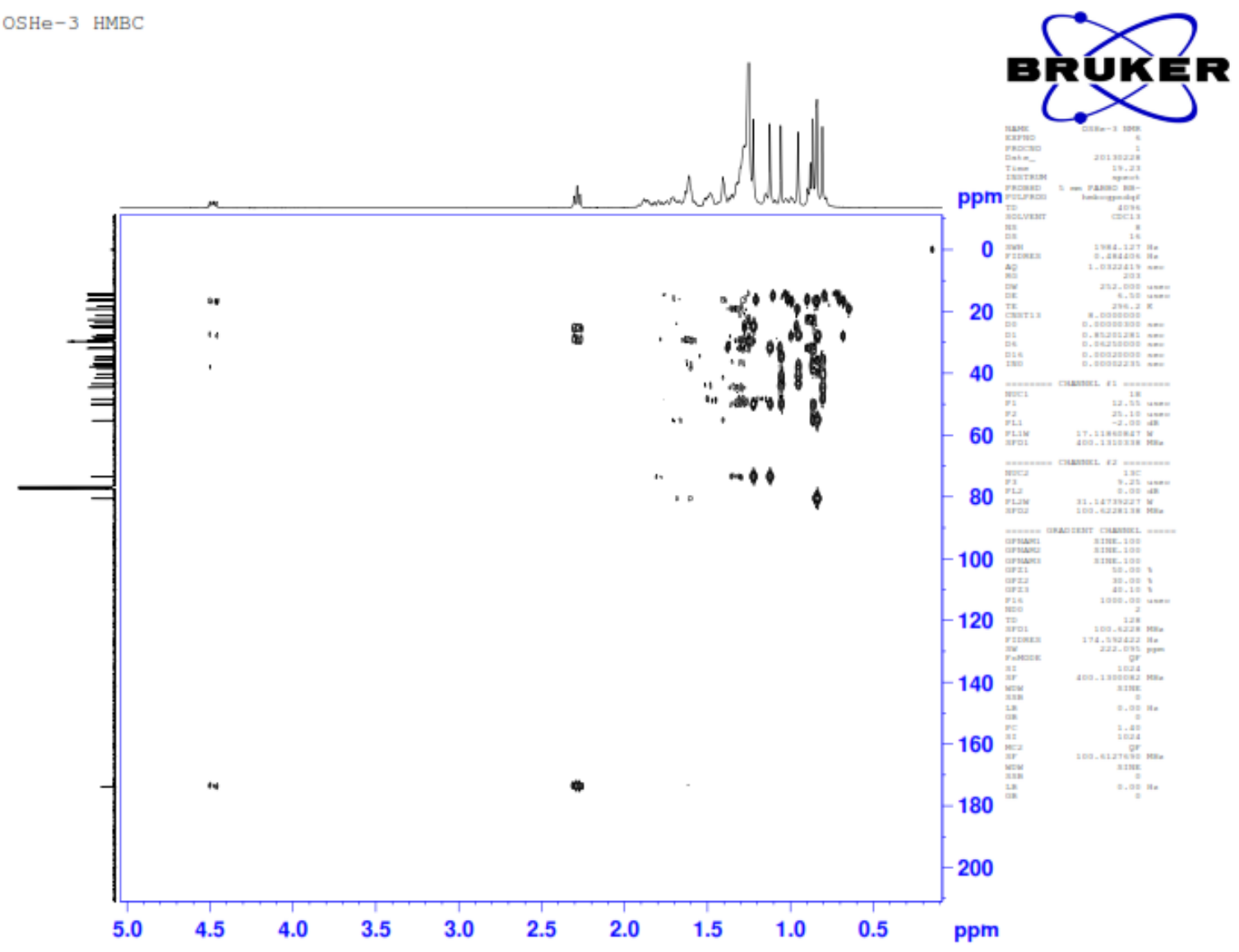




\section{HMBC EXPANSION 01}

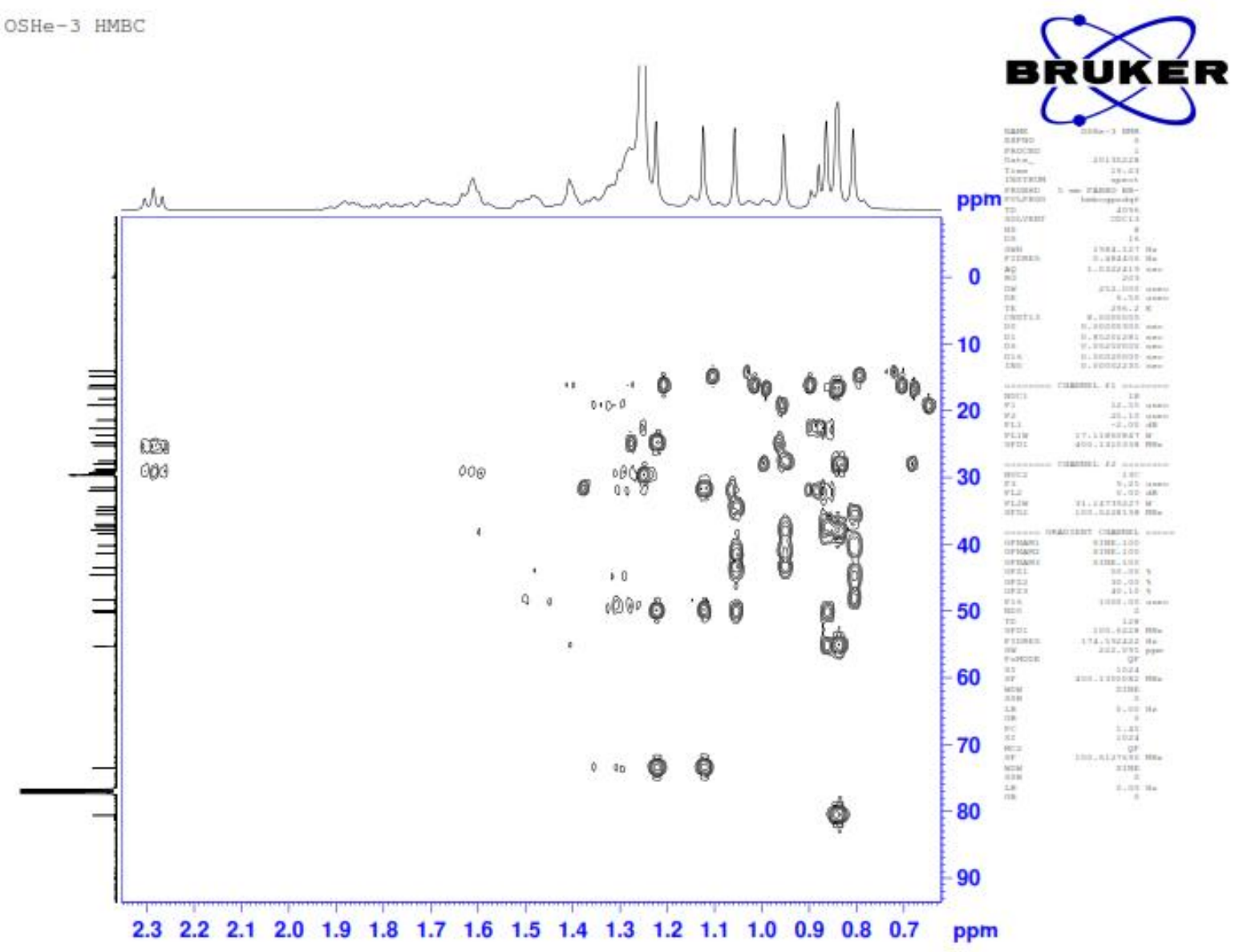


HSQC

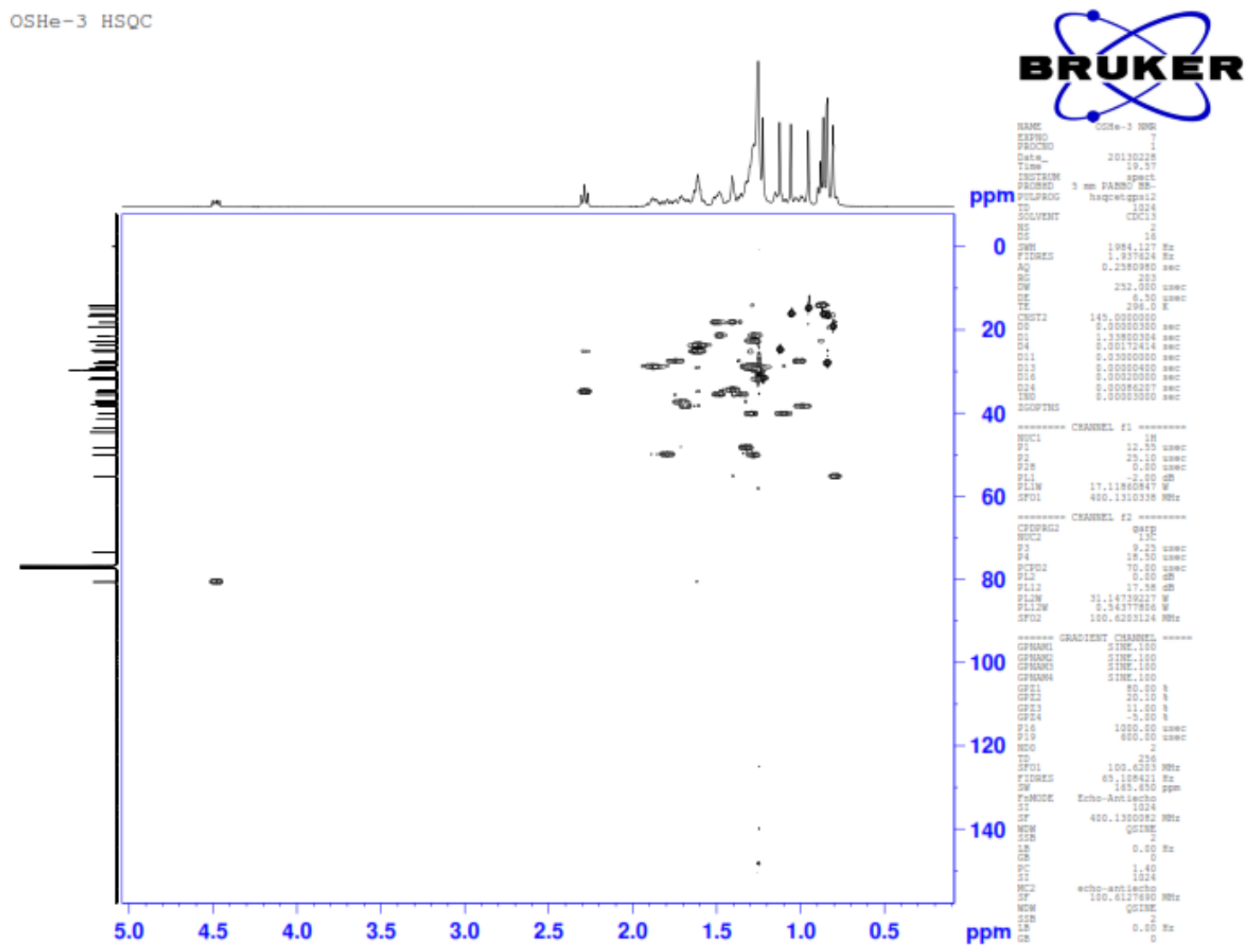




\section{HSQC EXPANSION 01}

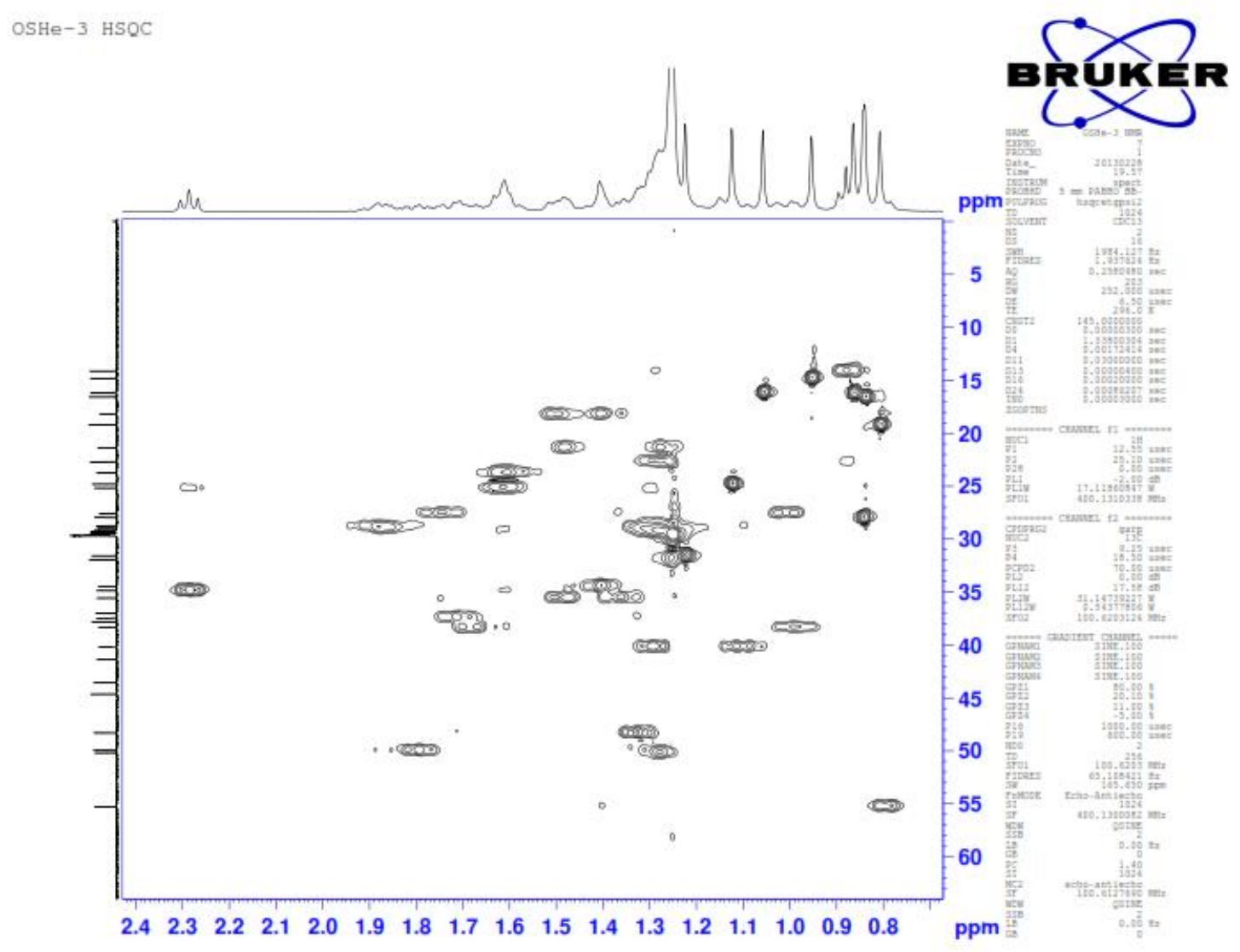




\section{NOESY}

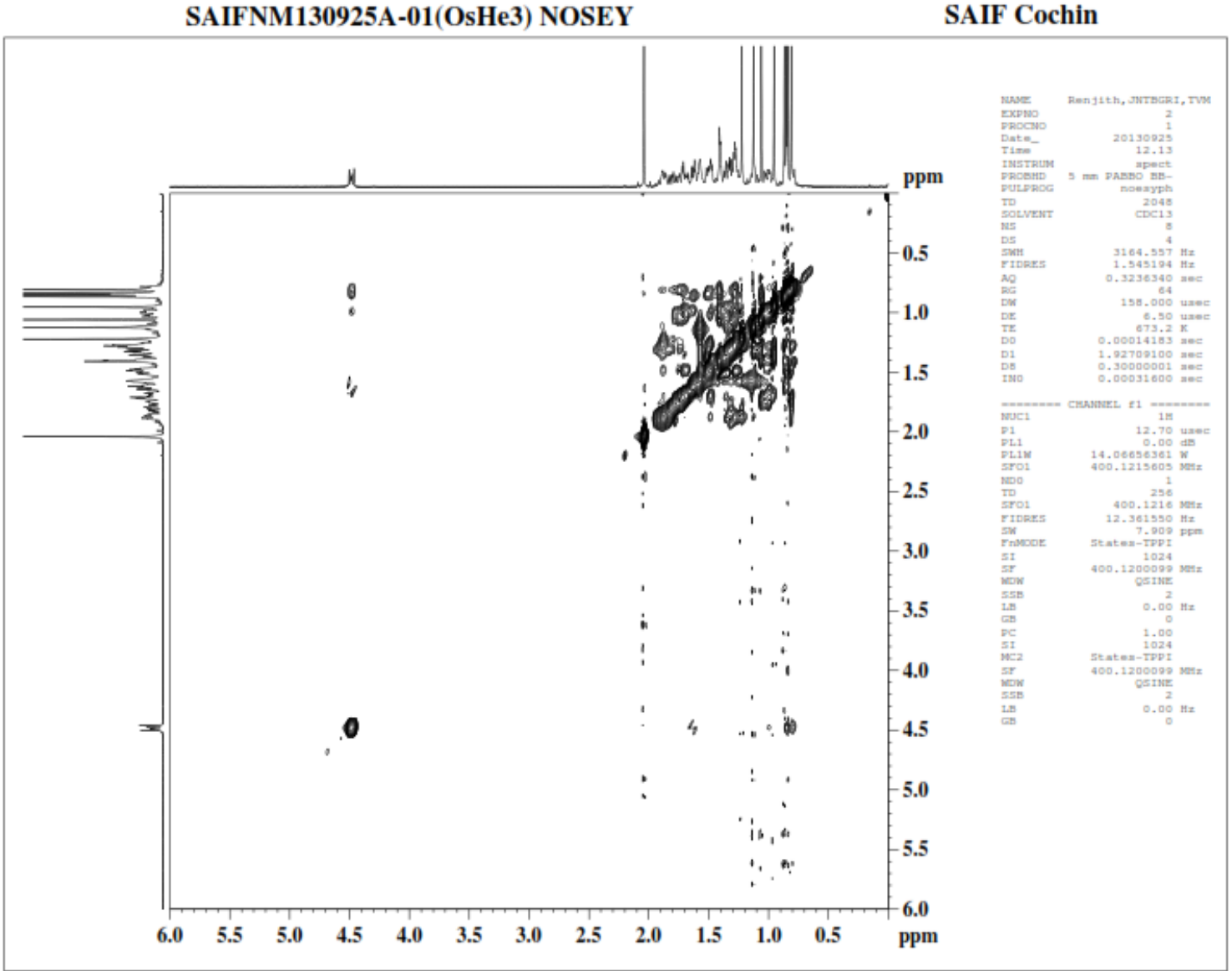




\section{NOESY EXPANSION 01}

SAIFNM130925A-01(OsHe3) NOSEY

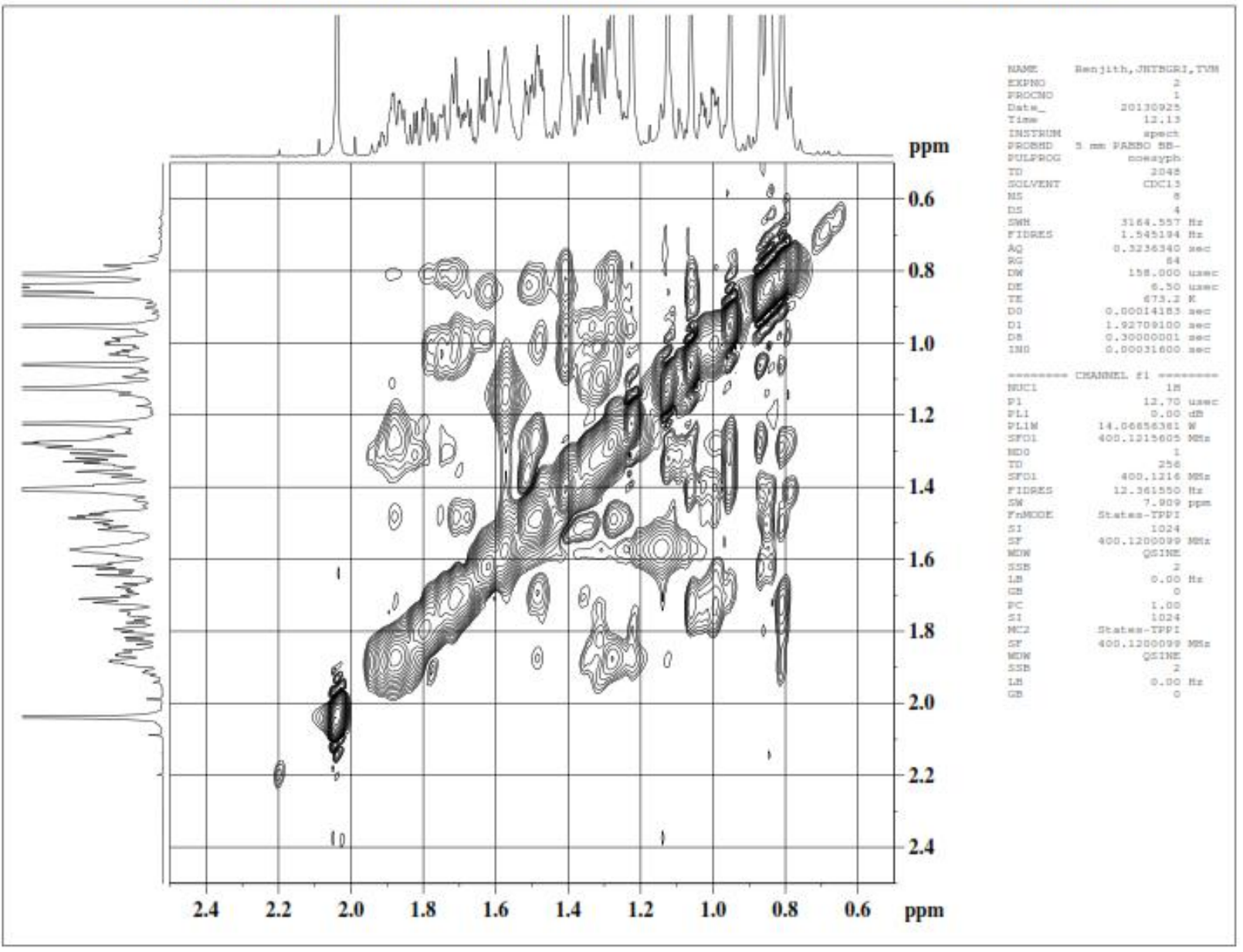




\section{MASS SPECTRUM}

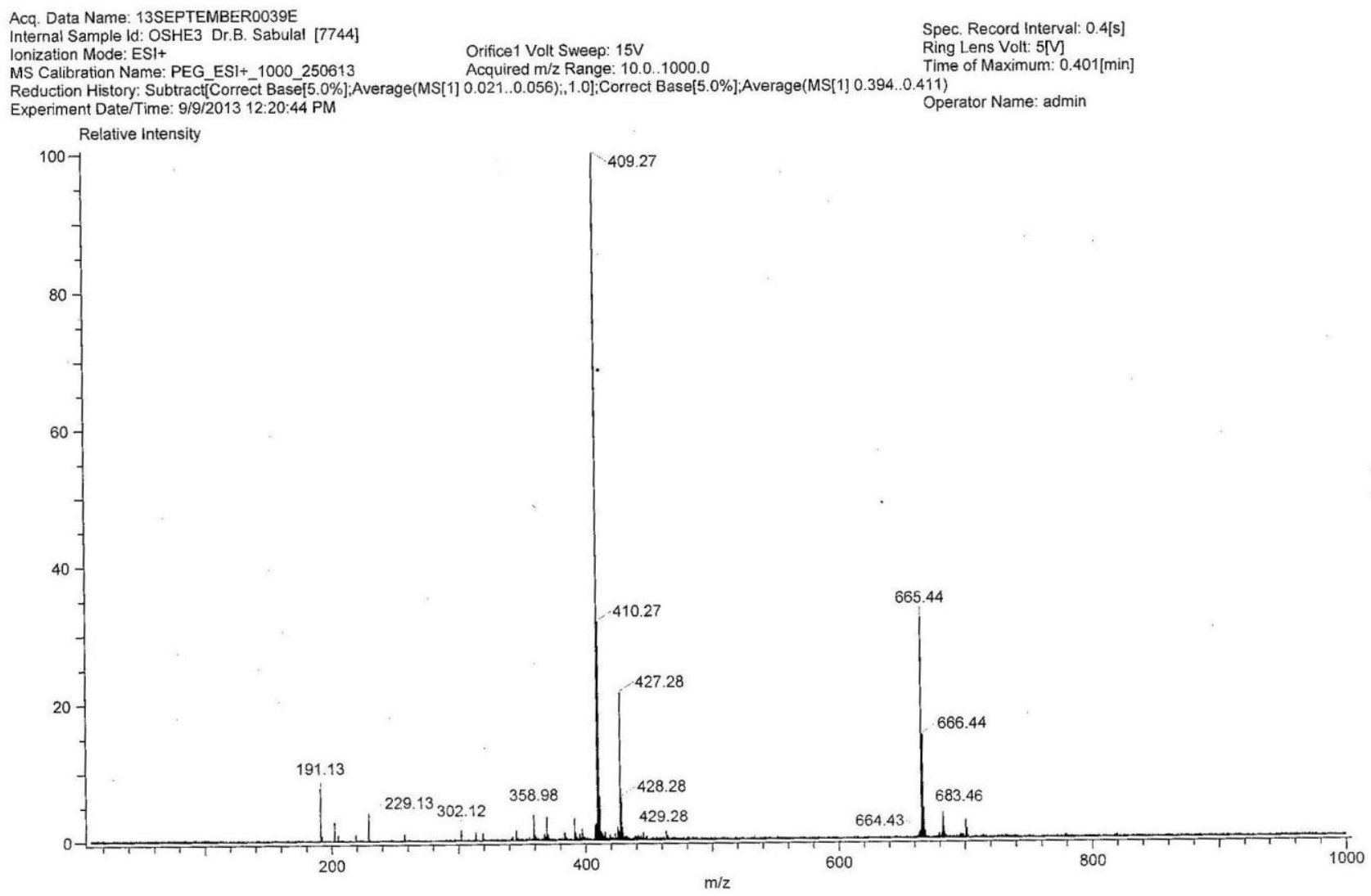




\section{GC-MS TIC}

File :C:Imsdchem|2IEOIL2013ไjulyłranjith|26071305.D

Operator :

Acquired : 26 Jul 2013 15:47 using AcqMethod ESSENTIALOIL_MANUAL700 M.M

Instrument: Instrument \#2

Sample Name: $\mathrm{OSHe} 3 \mathrm{H}$

Misc Info :

Vial Number: 1

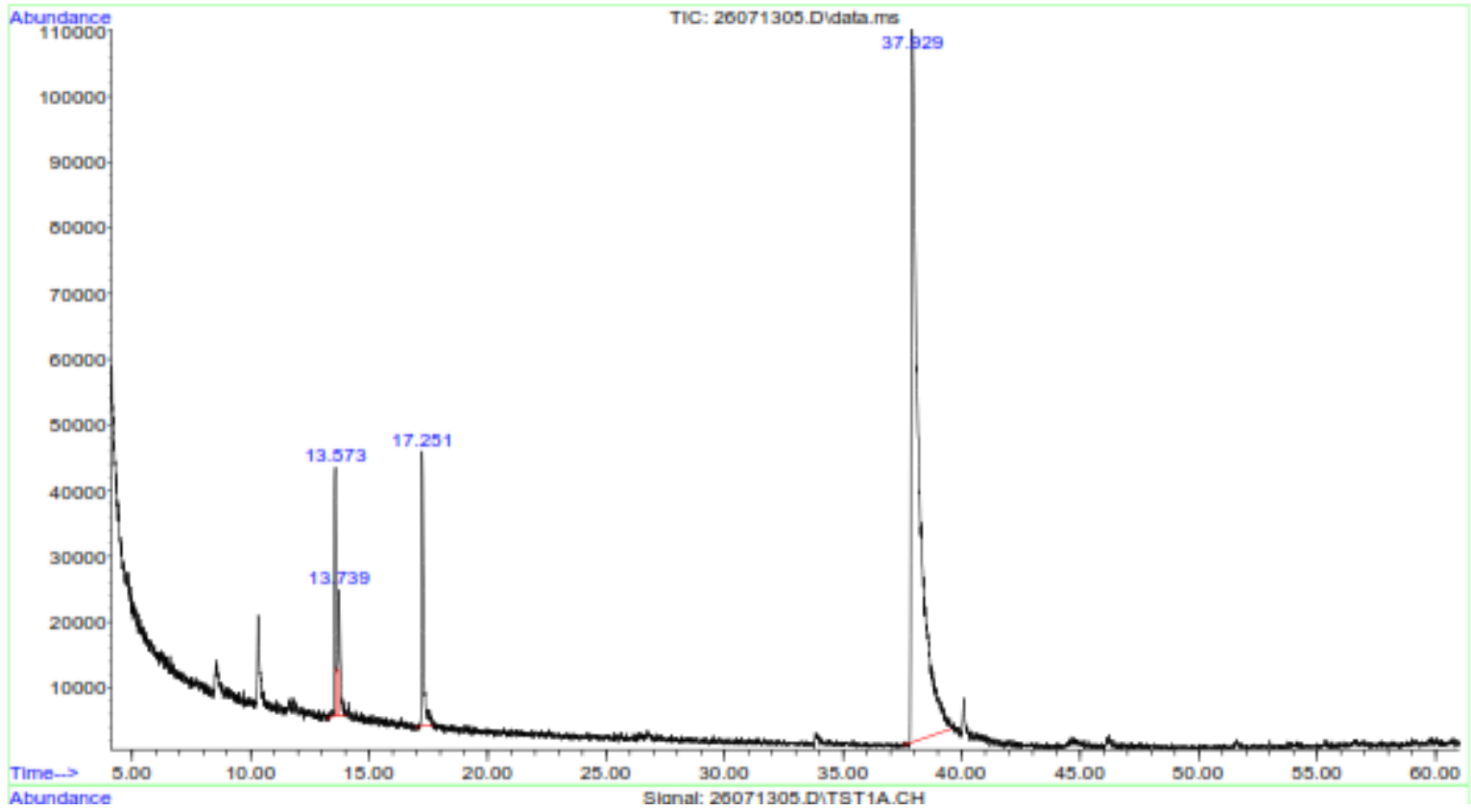


GC-MS MASS SPECTRUM (Methyl ester of hexadecanoic acid)

\section{* Search Report Page 1 of 1 *}

Unknown: Scan 4556 (37.932 min): 26071305 .Didata.ms

Compound in Library Factor $=199$

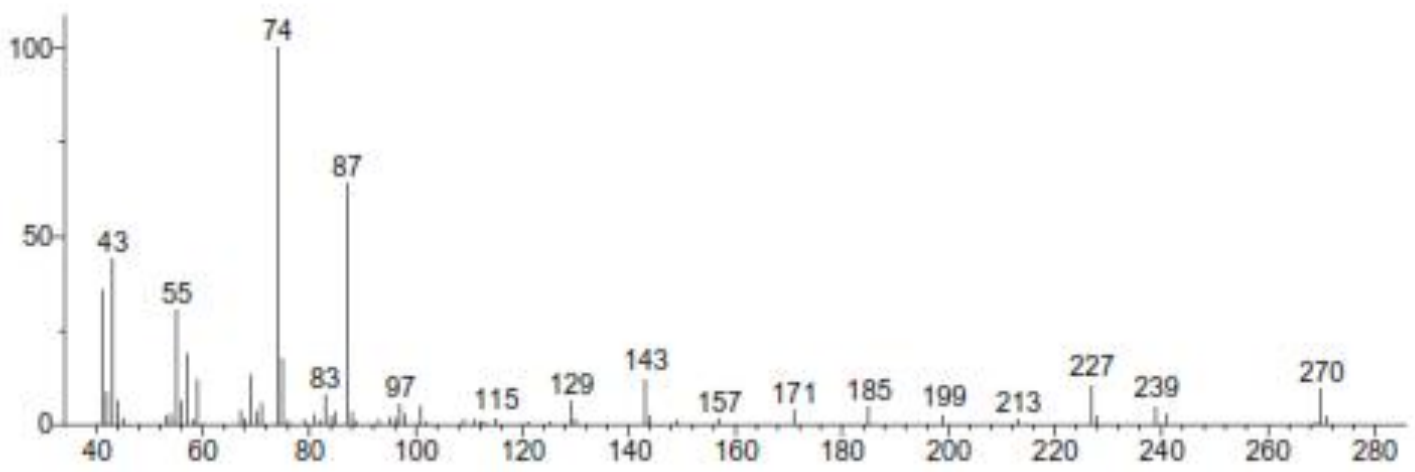

Hit 1 : Hexadecanoic acid, methyl ester

C17H3402; MF: 899; RMF: 903; Prob 69.7\%; CAS: 112-39-0; Lib: replib; ID: 9000.

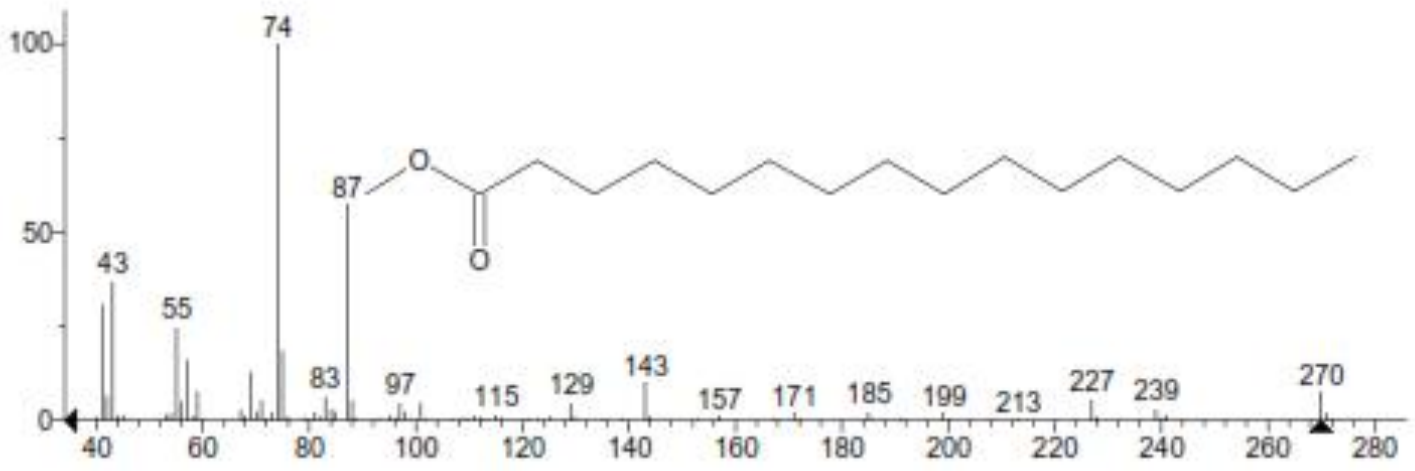


Spectral data of other isolated compounds ( 2 to 9 )

Lupan-20-ol-3( $\beta$ )-yl acetate (2): White amorphous solid; m. p. $245^{\circ} \mathrm{C}$; IR $\lambda_{\max } \mathrm{cm}^{-1}: 3494,2941$, 1714, 1455, 1370, 1263, 1190, 1160, 979; HRESI-MS m/z: $509.1899(\mathrm{M}+\mathrm{Na})^{+}$, calculated mass for $\mathrm{C}_{32} \mathrm{H}_{54} \mathrm{O}_{3} \mathrm{Na} 509.3970$; spectral data $\left({ }^{1} \mathrm{H},{ }^{13} \mathrm{C}\right.$ NMR, correlation data) of (2) were comparable with literature data (Yuruker et al. 1998). Compound (2), lupan-20-ol-3( $\beta$ )-yl acetate, has an acetate group at C3 instead of the long chain fatty acid ester side chain in (1) (Fig. 1). Spectral data of (2) were matching with literature (Yuruker et al. 1998) and also with the terpenoid skeleton of (1).

Olean-18-en-3( $\beta$ )-yl hexadecanoate (3): White waxy solid; m. p. $75^{\circ} \mathrm{C}$; IR $\lambda_{\max } \mathrm{cm}^{-1}: 2917,2850$, 1726, 1465, 1454, 1378, 1265, 1247, 1221, 1173, 981, 718; ESI-MS m/z: $663.47(\mathrm{M}+\mathrm{Na})^{+}$; spectral data $\left({ }^{1} \mathrm{H},{ }^{13} \mathrm{C}\right.$ NMR, correlation data) of (3) were comparable with Ragasa et al. 2012. Compound (3), olean-18-en-3( $\beta$ )-yl hexadecanoate (Fig. 1), has a double bond (C18, C19) and a long chain fatty acid side chain at C3 (Ragasa et al. 2012).

Dotriacontanoic acid (4): White amorphous solid; m. p. $95^{\circ} \mathrm{C}$; IR $\lambda_{\max } \mathrm{cm}^{-1}: 2915,2848,1737$, 1704, 1472, 1462, 1217, 729, 719; DART-MS $m / z: 481.36(\mathrm{M}+\mathrm{H}){ }^{+}$; spectral data $\left({ }^{1} \mathrm{H},{ }^{13} \mathrm{C} \mathrm{NMR}\right.$, correlation data) of (4) were comparable with Parmar et al., 1998.

Stigmasterol (5): Compound (5) (Fig. 1) was identified by comparison of its IR spectrum, m. p. and NMR data with literature (De-Eknamkul and Potduang 2003).

Rubiadin (6): Yellow solid; m. p. $291^{\circ} \mathrm{C}$; DART MS: $255.14\left(\mathrm{M}+\mathrm{H}^{+}\right)$; IR $\lambda_{\max } \mathrm{cm}^{-1}$ : 3390, 2921, 2515, 1659, 1619, 1579, 1548, 1503, 1479, 1394, 1335, 1300, 1133, 967, 710; ${ }^{13} \mathrm{C}$ NMR: 161.93 (C1), 119.93 (C2), 163.48 (C3), 108.07 (C4), 126.14 (C5), 134.04 (C6), 134.23 (C7), 127.02 (C8), 187.08 (C9), 183.22 (C10), 130.88 (4a), 132.42 (C8a), 110.03 (C9a), 133.08 (C10a), 8.14 $\left(\mathrm{CH}_{3}\right), 2.095\left(\mathrm{CH}_{3}\right), 7.154$ (C4), 7.810 (C5), 7.931 (C8), 8.189 (C6), 8.206 (C7) (Fig. 1).

Nonadecanoic acid (7): White solid; m. p. $68^{\circ} \mathrm{C}$; DART MS: $299.06\left(\mathrm{M}+\mathrm{H}^{+}\right)$; IR $\lambda_{\max } \mathrm{cm}^{-1}$ : 2956, 2915, 2848, 1695, 1471, 1429, 1411, 1347, 1330, 1295, 1187, 1099, 934, 720, ${ }^{13} \mathrm{C}$ NMR: 180.76, 
$34.21,24.73,29.79,29.77,29.74,29.69,29.53,29.46,29.33,29.15,32.02,22.77,14.15 ;{ }^{1} \mathrm{H}$ NMR: $2.34(\mathrm{t}), 1.6(\mathrm{~m}), 1.258(\mathrm{brs}), 0.879(\mathrm{t})$.

Hexadecanoic acid (Palmitic acid) (8): White solid; m. p. 63 ${ }^{\circ} \mathrm{C}$; DART-MS: $257.19\left(\mathrm{M}+\mathrm{H}^{+}\right)$; IR $\lambda_{\max } \mathrm{cm}^{-1}: 2955,2915,2848,1695,1463,1429,1411,1347,1330,1295,1187,1099,934,720$; ${ }^{13}$ C NMR: 180.41, 34.12, 31.96, 29.72, 29.70, 29.67, 29.39, 29.27, 29.09, 14.70, 22.72, 14.13; ${ }^{1} \mathrm{H}$ NMR: $2.346(\mathrm{t}), 1.63(\mathrm{~m}), 1.25-1.30(\mathrm{brs}), 0.88(\mathrm{t})$.

Camptothecin (9): Pale yellowish solid; m. p. $275^{\circ} \mathrm{C}$; LC-EI-MS, m/z: $349.52\left(\mathrm{M}+\mathrm{H}^{+}\right)$; IR $\lambda_{\max }$ $\mathrm{cm}^{-1}: 3430,2938,1737,1650,1600,1579,1499,1437,1349,1323,1233,1197,1156,1114$, 1040, 1005, 916, 829, 724; ${ }^{1} \mathrm{H}$ NMR (8): 5.28 (H-5), 8.66 (H-7), 8.10 (d, H-9, J = 8.0), 7.70 (t, $\mathrm{H}-10, \mathrm{~J}=7.2$ ), 7.85 (t, H-11, J = 7.6), 8.16 (d, H-12, J = 8.4), 7.37 (s, H-14), 5.41 (dd, H-17 J =

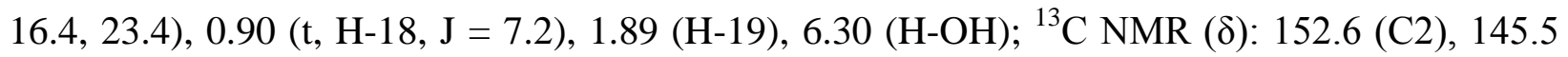
(C3), 50.1 (C5), 129.7 (C6), 131.5 (C7), 128.0 (C8), 128.4 (C9), 127.5 (C10), 130.26 (C11), 129.0 (C12), 148.1 (C13), 96.7 (C14), 150.0 (C15), 119.1 (C16), 156.8 (C16a), 65.3 (C17), 7.7 (C18), 30.7 (C19), 72.4 (C20), 172.2 (C21) (Fig. 1).

\section{References}

De-Eknamkul W, Potduang B. 2003. Biosynthesis of $\beta$-sitosterol and stigmasterol in Croton sublyratus proceeds via a mixed origin of isoprene units. Phytochemistry. 62:389-398.

Parmar VS, Jain SC, Gupta S, Talwar S, Rajwanshi VK, Kumar R, Azim A, Malhotra S, Kumar N, Jain R, Sharma NK, Tyagi OD, Lawrie SJ, Errington W, Howarth OW, Olsen CE, Singh, S. K., Wengel J. 1998. Polyphenols and alkaloids from Piper species. Phytochemistry. 49:1069-1078.

Ragasa CY, Espineli DL, Shen CC. 2012. A new triterpene from Barringtonia asiatica. Nat Prod Res. 26:1869-1875.

Yuruker A, Orjala J, Sticher O, Rali T. 1998. Triterpenes from Rhus taitensis. Phytochemistry. 48:863-866. 